\title{
Novel Modulation Detection Scheme for Underwater Acoustic Communication Signal Through Short-Time Detailed Cyclostationary Features
}

\author{
Xue $\mathrm{Li}^{1}$, Qian $\mathrm{Han}^{2}$, Zhiqiang $\mathrm{Liu}^{3}$ and Zhiqiang $\mathrm{Wu}^{4}$ \\ Mobile Solutions Lab, Samsung Inf. Syst. America Inc. ${ }^{1}$ \\ Financial Advisory Services, Deloitte ${ }^{2}$ \\ Acoustic Division, Naval Research Laboratory ${ }^{3}$ \\ Department of Electrical Engineering, Wright State University ${ }^{4}$
}

\begin{abstract}
In this paper, we propose a new method for blind modulation detection based on detailed second order cyclostationary features for underwater acoustic communication. Due to the severe and fast varying Doppler shift and phase noise in the underwater acoustic communication channel, much shorter signal length can be used to detection the modulation scheme, leading to less accuracy in the modulation detection performance. Here we propose a brand new method to perform modulation detection: by analyzing the zoomed-in spectral coherence function (SOF) of short time signal, we observe distinctive detailed features for different modulations. Taking advantage of such detailed features in short time SOF, we design a novel blind modulation detection algorithm for underwater acoustic communication system. To the best of our knowledge, our paper is the first to employ such detailed, in some sense hidden, features of cyclostationary analysis to conduct modulation detection. Coupled with our previous work on blind carrier frequency estimation and symbol rate estimation algorithms, the proposed modulation detection algorithm enjoys very high detection accuracy, high speed, and low complexity. Real experimental data collected at sea are used to validate the effectiveness of the proposed algorithm. It is also important to note that the proposed method does not assume any a priori knowledge of the target signal.
\end{abstract}

\section{INTRODUCTION}

Cyclostationary analysis has been accepted as an important tool to perform signal detection, signal parameter estimation, and modulation detection of radio frequency (RF) signals [1] [8]. Cyclostationary analysis is based on the fact that communications signals are not accurately described as stationary, but rather more appropriately modeled as cyclostationary. While stationary signals have statistics that remain constant in time, the statistics of cyclostationary signals vary periodically. These periodicities occur for signals of interest in well defined manners due to underlying periodicities such as sampling, modulating, multiplexing, and coding. This resulting periodic nature of signals can be exploited to detect the existence of the signal, estimate important parameters of the signal, and determine the modulation scheme of the unknown signal.

However, due to the extremely complex and dynamic environment of underwater acoustic communication, it is not clear if the cyclostationary analysis is still applicable in these tasks.
The underwater acoustic channels are extremely complex and dynamic [9]-[12]. The underwater acoustic communication signals experience severe Doppler shift, multi-path effect, phase noise and variation over time. As a direct result, the cyclostationary analysis faces significant challenges in analyzing underwater acoustic communication signals.

As shown previously, the Doppler shift for a moving source can vary rapidly with time [12], and the multi-path effect also changes very quickly with time. As a direct result, the conventional analysis using the second order spectral correlation function $(\mathrm{SCF})$ or spectral coherence function (SOF), which treats the channel as time invariant, does not always work. To estimate the fast varying Doppler shift, one must apply the cyclostationary analysis to shorter segments of the signal where the channel can be assumed static. However, by shorten signals in smaller segments, traditional second order cyclostationary features are significantly distorted, leading to large performance degradation in modulation detection. In our previous works, we have applied cyclostationary analysis to underwater acoustic communication signals and developed blind carrier frequency (and Doppler shift) estimation, symbol rate estimation [13], and modulation detection algorithms [14]. However, while the blind carrier frequency estimation and symbol rate estimation offer high accuracy in the parameter estimation, the modulation detection results are not ideal, with the detection accuracy varying between $90 \%$ to $92 \%$.

In this paper, we propose a brand new method for blind modulation detection of underwater acoustic communication signals. By analyzing the zoomed-in spectral coherence function of received underwater acoustic communication signals after transmission through complex underwater channels, we observe distinctive features for different modulation types. Taking advantage of such detailed features, we design a simple yet effective modulation detection algorithm. This low complexity algorithm provides highly accurate classification performance for the underwater acoustic communication systems, with modulation detection accuracy higher than $96 \%$. Real data collected at sea is used to confirm the effectiveness, 
fast speed and low complexity of the proposed algorithm. To the best of our knowledge, our paper is the first to employ such detailed, in some sense hidden, features of cyclostationary analysis to conduct modulation detection, not only for underwater acoustic communication signals, but for RF signals as well.

The rest of the paper is organized as follows: In section II, we present the underwater acoustic communication signal model and the cyclostationary analysis. Section III describes the signal classification algorithm based on detailed SOF. Section IV shows numerical results of the signal modulation classification.

\section{System Model and Cyclostationary ANAlysis}

A. System Model of Underwater Acoustic Communication Signal

The transmitted signal of a single carrier underwater communication system corresponds to

$$
s(t)=\sum_{i=-\infty}^{\infty} b_{i} p(t-i T) \cos \left(2 \pi f_{c} t\right)
$$

where $b_{i}$ is the $i^{\text {th }}$ data symbol $\left(b_{i} \in\{+1,-1\}\right.$ for BPSK modulation), $p(t)$ is the pulse shape, $T$ is the symbol duration, and $f_{c}$ is the carrier frequency. At receiver side, the received signal experiences multi-path fading, Doppler shift, phase noise, and additive white Gaussian noise:

$$
\begin{array}{r}
r(t)=\Re\left\{\sum_{l=0}^{L-1} \sum_{i=-\infty}^{\infty} \alpha_{l}(t) e^{j \theta_{l}(t)} b_{i} p\left(t-i T-\tau_{l}(t)\right)\right. \\
\left.\cdot e^{j 2 \pi\left(f_{c}+\Delta f(t)\right)\left(t-\tau_{l}(t)\right)}\right\}+n(t)
\end{array}
$$

where $L$ is the maximum number of multi-path components, $l$ is the index for multi-path component, $\alpha_{l}$ is the fading gain of the $l^{t h}$ path, $\theta_{l}$ is the phase offset introduced by the channel on the $l^{t h}$ path, $\tau_{l}$ is the time delay of the $l^{t h}$ path, $\Delta f$ is the Doppler shift, and $n(t)$ corresponds to the additive white Gaussian noise. Note that the fading, Doppler shift and phase noise are all functions of time.

If we calculate the second order cyclostationary features of the underwater acoustic communication signal over a long period of time, the varying multi-path fading, Doppler shift and phase noise would distort the cyclic frequency features, making the those features meaningless. Hence, we have to apply a time domain window onto the received signal to obtain a short length of received signal so that within this length the channel and Doppler shift can be considered stable. Mathematically, this truncated signal corresponds to

$$
\begin{aligned}
r_{0}(t)= & r(t) \cdot w(t) \\
= & \Re\left\{\sum_{\substack{l=0 \\
L-1}}^{\infty} \sum_{i=-\infty}^{\infty} \alpha_{l} e^{j \theta_{l}} b_{i} p\left(t-i T-\tau_{l}\right)\right. \\
& \left.\cdot e^{j 2 \pi\left(f_{c}+\Delta f\right)\left(t-\tau_{l}\right)}\right\}+n(t), \quad 0 \leq t \leq T_{w}
\end{aligned}
$$

where $T_{w}$ is the length of the rectangular window $w(t)$. It is generally agreed that the underwater channel can be assumed stable within 0.1 to 0.2 seconds. In this article we assume the length $T_{w}$ is 0.128 second.

\section{B. Cyclostationary Analysis and SCF/SOF}

It is well known that most of communication signals are cyclostationary, and cyclostationary features such as spectral correlation function (SCF) and spectral coherence function (SOF) can be used in signal detection, parameter estimation and modulation detection [2]. Compared to the SCF $S_{X}^{\alpha}(f)$, as a normalized version of the SCF, the SOF can help remove the channel effect:

$$
C_{X}^{\alpha}(f)=\frac{S_{X}^{\alpha}(f)}{\left[S_{X}^{0}\left(f+\frac{\alpha}{2}\right)^{*} S_{X}^{0}\left(f-\frac{\alpha}{2}\right)\right]^{1 / 2}}
$$

For BPSK modulated signal $x=a(t) \cos \left(2 \pi f_{c} t+\phi_{0}\right)$, the spectrum correlation function can be expressed as [2]:

$$
S_{x}^{\alpha}(f)=\left\{\begin{array}{cc}
\frac{1}{4}\left[S_{a}^{0}\left(f-f_{c}\right)+S_{a}^{0}\left(f+f_{c}\right)\right] & \alpha=0 \\
\frac{1}{4} e^{j 2 \phi_{0}} S_{a}^{0}(f) & \alpha=2 f_{c} \\
\frac{1}{4} e^{-j 2 \phi_{0}} S_{a}^{0}(f) & \alpha=-2 f_{c} \\
0 & \text { others }
\end{array}\right.
$$

where $\alpha$ represents the cyclic frequency, and $S_{a}^{0}(f)$ is the Fourier transform of the autocorrelation $R_{a}^{0}(\tau)$, if rectangular pulse shaping is applied:

$$
S_{a}^{0}(f)=\text { Fourier }\left[R_{a}^{0}(\tau)\right]=T_{b} \operatorname{sinc}^{2}\left(f T_{b}\right)
$$

where $T_{b}$ denotes the symbol duration.

For QPSK and higher modulated signal, due to the symmetric property, the spectrum correlation function can be expressed as [2]:

$$
S_{x}^{\alpha}(f)=\left\{\begin{array}{cc}
\frac{1}{4}\left[S_{a}^{0}\left(f-f_{c}\right)+S_{a}^{0}\left(f+f_{c}\right)\right] & \alpha=0 \\
0 & \text { others }
\end{array}\right.
$$

Fig. 1(a) and (b) illustrate the theoretical SCFs of BPSK and QPSK modulated signals. From these figures, we can observe that there are four peaks in the frequency-cyclic frequency domain for BPSK modulated signal. Two of these peaks appear in the frequency axis where $\alpha=0$ and $f= \pm f_{c}$, and other two appear in the cyclic frequency axis where $f=0$ and $\alpha= \pm 2 f_{c}$. It is obvious that when $\alpha=0$ the SCF reduces to the power spectrum density (PSD) of the signal. For the QPSK modulated signal, only two peaks appear in the frequency axis where $\alpha=0$ and $f= \pm f_{c}$.

Because of this difference in the SCF/SOF of BPSK and QPSK (and higher PSK and QAM modulation schemes), the $\mathrm{SCF} / \mathrm{SOF}$ is often used to distinguish the modulation scheme of a RF signal. It is important to note that Fig. 1 shows the theoretical SCFs which come from infinite length of data. In reality, we can only obtain a limited length of signal to perform SCF.

\section{SCF of Short Time Underwater Acoustic Signal}

However, since we can only obtain the SCF/SOF of the underwater acoustic signal from much shorter length of signal segments (Eq. (3)), the difference between the SCF/SOF of a 


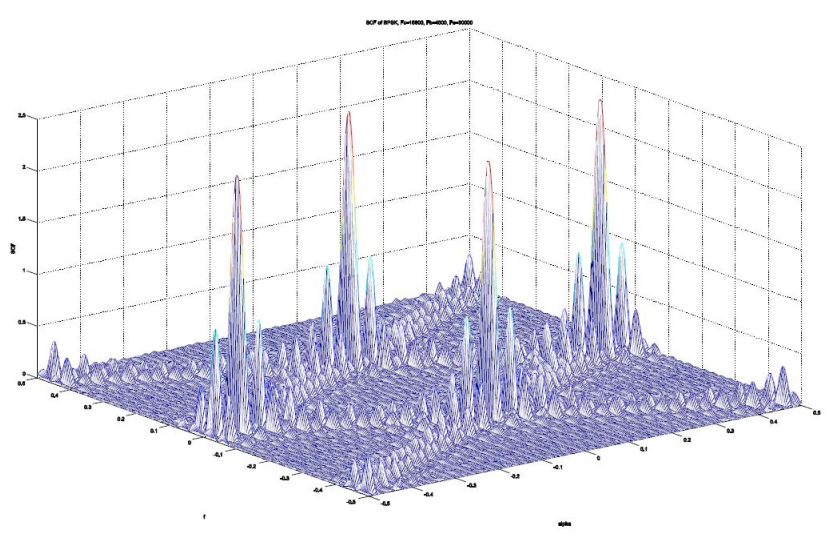

(a) BPSK Modulation

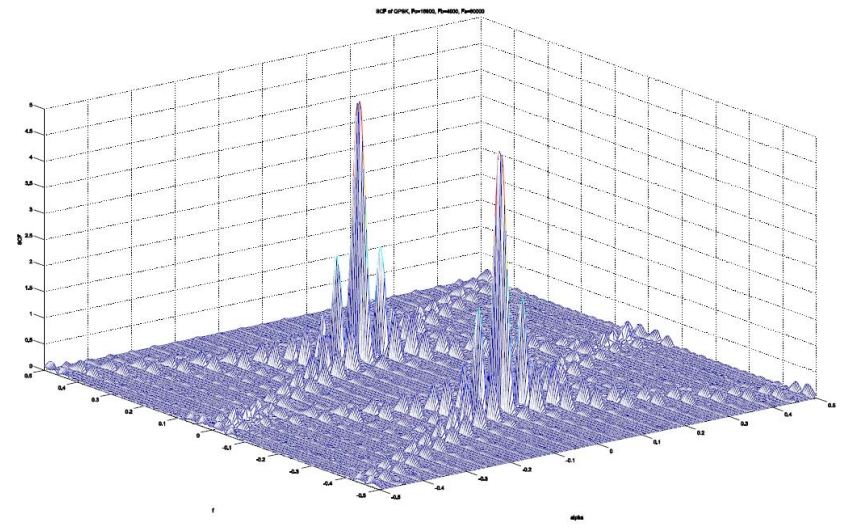

(b) QPSK Modulation

Fig. 1. Spectral Correlation Function

BPSK signal and that of a QPSK (and higher modulations) signal diminishes. Fig. 2 illustrate the SCF 3-D plots of short time BPSK signal and QPSK signal in underwater acoustic communication. Unlike the SCF obtained from very long time signal in Fig. 1, it is now difficult to notice the difference between these two SCFs. Hence, traditional modulation detection based on the existence of peaks at $\alpha= \pm 2 f_{c}, f=0$ does not work well in the underwater acoustic communication systems [14].

\section{Blind Modulation Detection}

Now we propose a brand new method to conduct modulation detection by using some detailed second order cyclostationary features never been used before. We now zoom in the SOFs of different modulation type signals around the cyclostationary peaks in the cyclic-frequency domain. In other words, we want to observe the details of the little peaks on the cyclic frequency domain shown in Fig. 2. To achieve this, we first employ our previous developed blind parameter estimation algorithm to estimate the carrier frequency $f_{c}$ and symbol rate $f_{b}$, then observe the the SOF around twice the carrier frequency in the cyclic frequency domain. Fig. 3 shows the block diagram of the whole blind estimation/modulation detection setup.

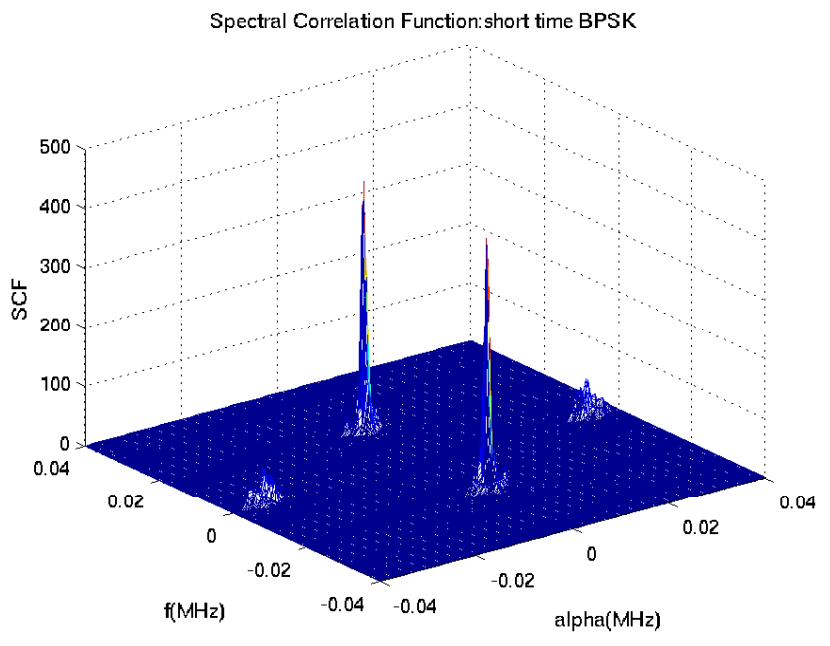

(a) BPSK Modulation

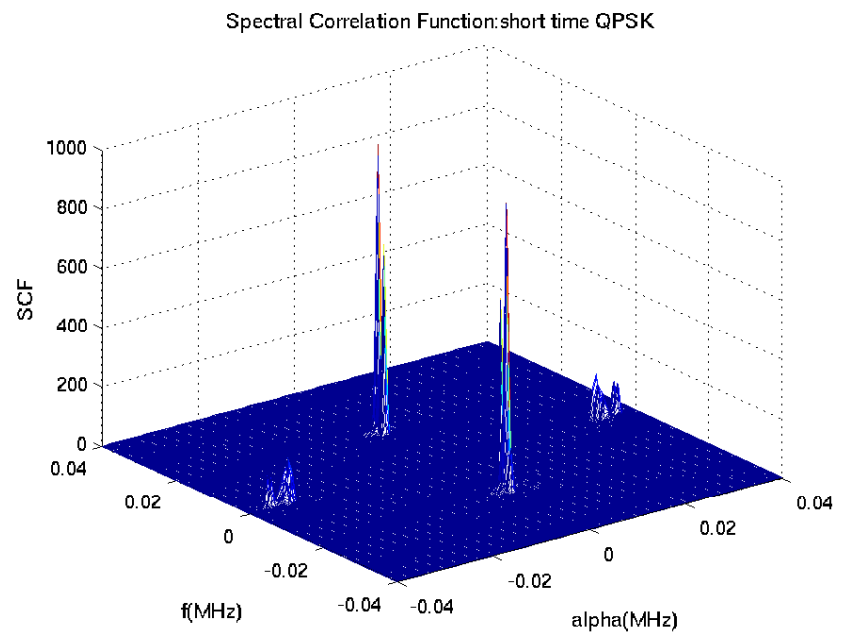

(b) QPSK Modulation

Fig. 2. Short Time SCF of Underwater Acoustic Signals

Fig. 4 shows the top-down view of zoomed-in SOF of BPSK/QPSK/16QAM modulated underwater acoustic signals from real data collected at sea where the carrier frequency is $f_{c}=17000 \mathrm{~Hz}$ for all modulations, the symbol rate is $f_{b}=4000 \mathrm{~Hz}$ for QPSK and 16QAM and $f_{b}=5000 \mathrm{~Hz}$ for BPSK. The $\mathrm{x}$-axis indicates the cyclic frequency $\alpha$, and the $\mathrm{y}$ axis denotes the frequency $f$. Note that we are only observing $400 \mathrm{~Hz}$ around the center in the cyclic frequency domain (the center is at $\alpha=2 \cdot f_{c}$ ).

It is now clear that the detailed SOFs have distinctive differences for different modulation types: the energy for BPSK signal is concentrated in the low frequency range, while the energy for QPSK and 16QAM signals is concentrated on high frequency range. It is interesting to note that the detailed SOF of BPSK and that of QPSK (and higher modulations) are kind of complementary to each other. Specifically, when $\alpha$ is around $2 \cdot f_{c}$, BPSK has different SOF values at region $1\left(f \in\left[-f_{b}, f_{b}\right]\right)$ and at region $2\left(f \in\left[2 f_{c}-f_{b}, 2 f_{c}+f_{b}\right]\right)$, 


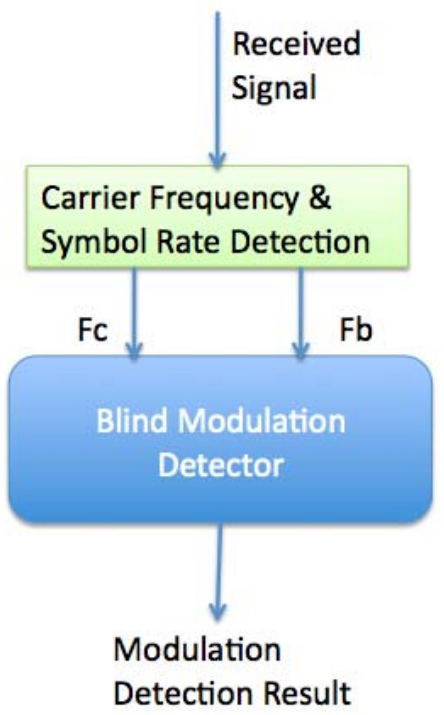

Fig. 3. Observing detailed features around cyclic peaks

i.e., region 1 has higher energy than region 2; while other modulations have similar peak in both regions. So instead of using the peak difference in region 1 like traditional SOF/SCF based modulation detection, the peak ratio in these two region provides more significant difference. As a direct result, the value ratio between these two regions can be applied as the metric to distinguish BPSK modulation from other higher order modulations.

To distinguish the difference between different modulations, we propose a low complexity algorithm:

1) Observe SOF around cyclic frequency of twice the carrier frequency, denoted as $S O F$ : carrier frequency can be estimated using some algorithm, e.g., we proposed an RF parameter estimation algorithm in [13];

2) Use rectangular window to smooth the absolute value of $S O F$, denoted as $S O F 2$;

3) Compute the maximal value of $S O F 2$ in the region $f \in$ $\left[-f_{b}, f_{b}\right]$, denoted as $M_{a x}$

4) Compute the maximal value of SOF2 in the region $f \in$ $\left[2 f_{c}-f_{b}, 2 f_{c}+f_{b}\right]$, denoted as $\operatorname{Max}_{2}$;

5) Compute the ratio:

$$
\text { Ratio }=\frac{\operatorname{Max}_{1}}{\operatorname{Max}_{2}}
$$

6) Classify the modulation using threshold TH:

$$
\text { Modulation }=\left\{\begin{array}{cl}
B P S K & \text { Ratio }>T H \\
\text { higher modulation } & \text { Ratio } \leq T H
\end{array}\right.
$$

By only observing the SOF around twice carrier frequency, and only requires the SOF in region 1 and region 2, the computation complexity is significantly reduced. From Figs. 2 and 4, it is clear that the threshold should be set to larger than 1 , which will be discussed in next section.

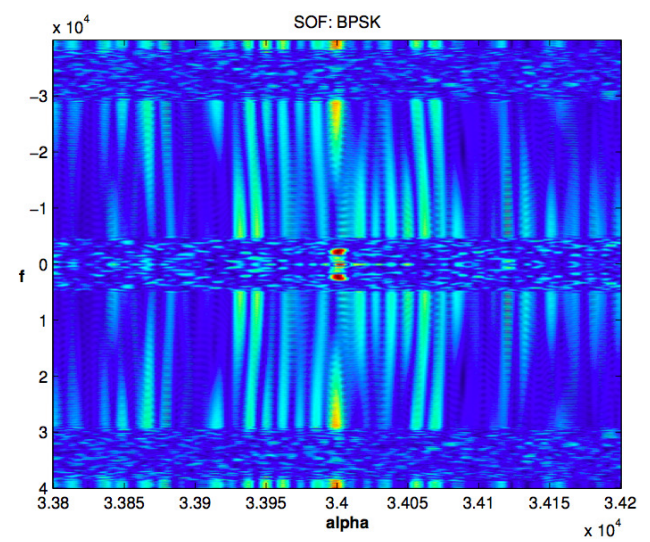

(a) BPSK Modulation

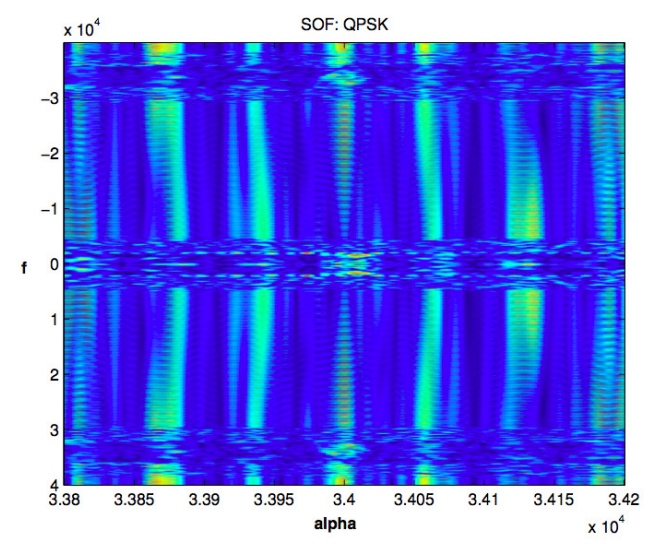

(b) QPSK Modulation

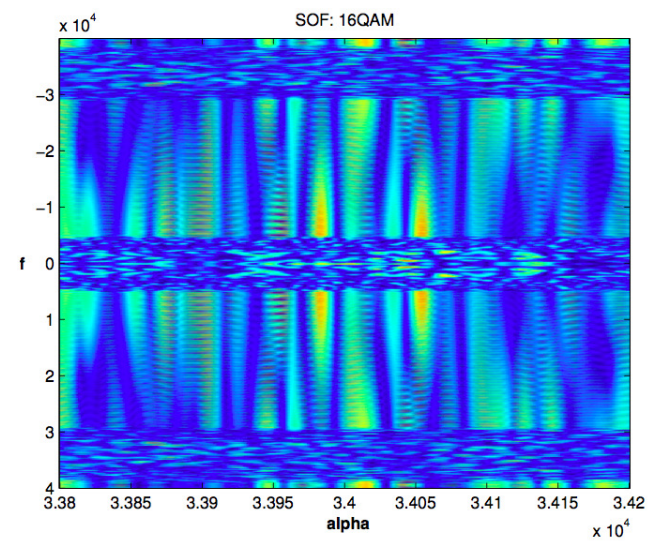

(c) 16QAM Modulation

Fig. 4. SOF at Cyclic Frequency around Carrier Frequency

\section{NumericAl RESUlts}

Now we use real underwater acoustic communication signal data collected at sea [12] to validate the effectiveness of the proposed algorithm. The transmitted signal has a carrier frequency of $f_{c}=17,000 \mathrm{~Hz}$ and symbol rate of $f_{b}=$ $4,000 \mathrm{~Hz}$ (QPSK and 16QAM) or $f_{b}=5,000 \mathrm{~Hz}$ (BPSK), the sampling rate is $f_{s}=80,000 \mathrm{~Hz}$. Considering the time varying channel and Doppler shift, the received signal is truncated into segments with 10240 samples in each segment, 
which is 0.128 seconds.

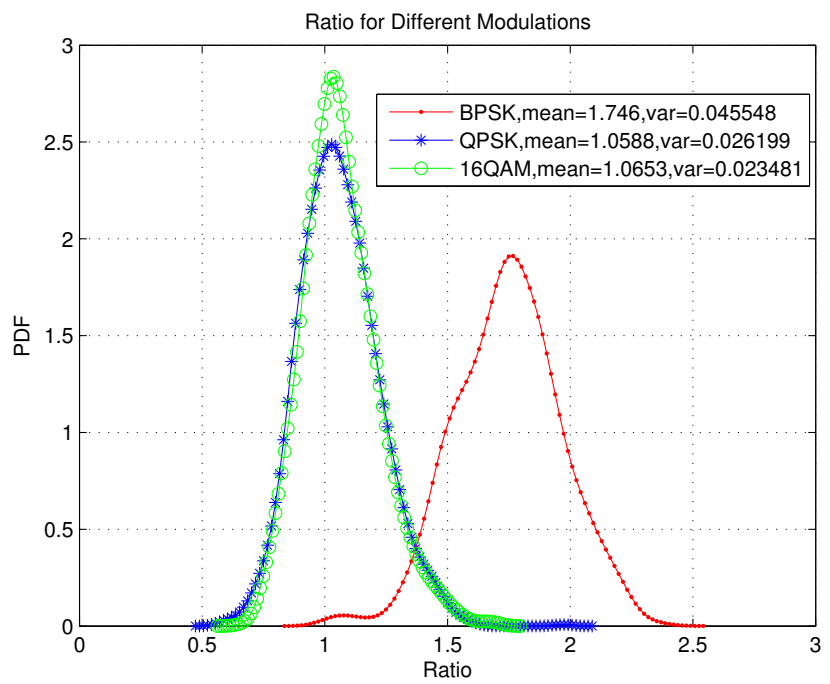

Fig. 5. PDF of Ratios for different modulations

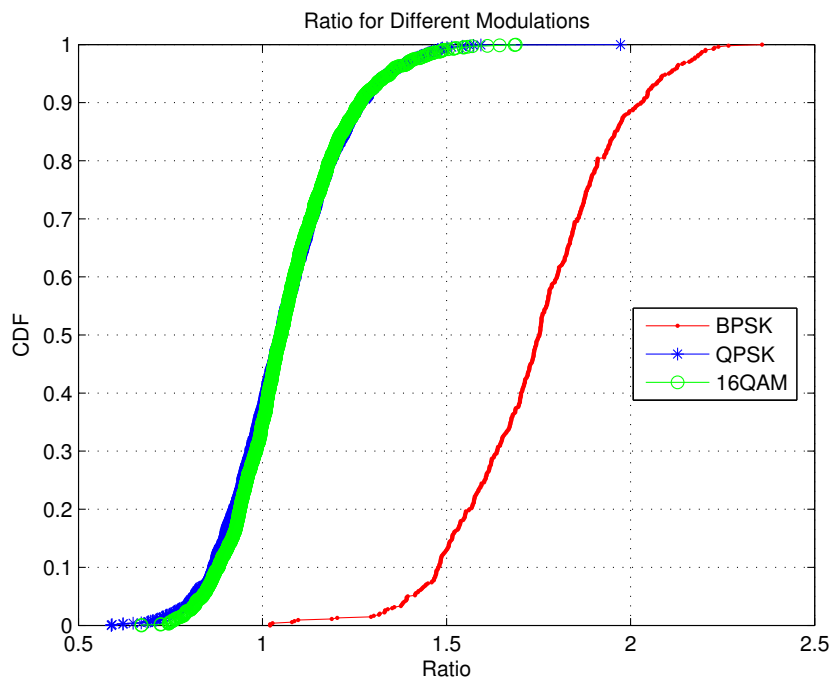

Fig. 6. CDF of Ratios for different modulations

To illustrate the feasibility of the proposed algorithm and search the optimal threshold, we analyze the Ratio in Eq. (8) for different modulations of underwater acoustic signals. Fig. 5 and Fig. 6 illustrate the PDF (probability density function) and CDF (Cumulative Distributed Function) of Ratio for different signals, segments and modulations. BPSK has higher Ratio since region 1 contains larger peak than region 2 in detailed SOF, e.g., in Fig. 4(a); QPSK and 16QAM has similar PDF and $\mathrm{CDF}$ of the Ratio, which is centered at 1 since similar peaks happen in both region 1 and region 2, e.g., in Fig. 4(b) and (c). The intersect point of PDF for BPSK and the PDF for QPSK/16QAM can be used as the threshold

$$
T H=1.37
$$

For different modulations, we have the mean and standard deviation of the Ratio:

$$
\begin{gathered}
E[\text { Ratio } \mid \text { BPSK }]=1.746 ; \\
\operatorname{Var}[\text { Ratio } \mid \text { BPSK }]=0.046 \\
E[\text { Ratio } \mid Q P S K]=1.059 ; \\
\operatorname{Var}[\text { Ratio } \mid \text { BPSK }]=0.026 \\
E[\text { Ratio } \mid 16 Q A M]=1.065 ; \\
\operatorname{Var}[\text { Ratio } \mid \text { BPSK }]=0.023
\end{gathered}
$$

To observe the modulation detection performance of the proposed algorithm, different signal data files from underwater acoustic communication systems are applied for each modulation, and each signal file is truncated into multiple segments. Specifically, BPSK modulated signals are observed from file TREX_M10_Fb5k_2004114060021_CH1.mat and so on; QPSK modulated signals are observed from file ACDS2 QPSK 1xI 2009145032840.mat and so on; 16QAM modulated signals are observed from file ACDS2_QAM16_1x1_2009145031711.mat and so on. In the simulation, 500 segments from different files are examined for BPSK modulated signals, while 2700 segments from different files are examined for QPSK and higher modulated signals.

By using the threshold in Eq. (10), underwater acoustic signals with different modulations are classified by the proposed modulation classification algorithm, and the classify results are shown in Table I. For received BPSK modulated short time signal, $96.5 \%$ are classified as BPSK modulation while only $3.5 \%$ are classified as non-BPSK modulation; for received QPSK and higher modulated short time signal, 96.4\% are classified as non-BPSK modulation while only $3.6 \%$ are classified as BPSK modulation. It is important to note that the real captured underwater signal in this paper experiences real underwater channel, including fading, Doppler shift, phase noise as well as additive white Gaussian noise.

TABLE I

Classification Performance for Underwater Signal

\begin{tabular}{|c|c|c|}
\hline Modulations & $\begin{array}{c}\text { Classify as } \\
\text { BPSK }\end{array}$ & $\begin{array}{c}\text { Classify as } \\
\text { Non-BPSK }\end{array}$ \\
\hline BPSK & $96.5 \%$ & $3.5 \%$ \\
\hline QPSK & $3.6 \%$ & $96.4 \%$ \\
\hline 16QAM & $3.6 \%$ & $96.4 \%$ \\
\hline
\end{tabular}

It is important to note that the test metric Ratio in Eq. (8) does not have the power uncertainty problem. For example, traditional cyclostationary detector utilizes the existence of the peak in cyclic frequency domain to distinguish BPSK and higher order modulation, and the peak value varies according to different signal power, e.g., large power produces higher peak. In our proposed detail SOF ratio detector, the Ratio will not be affected by the signal power: higher signal power will increase same level to region 1 peak and region 2 peak, 
hence the Ratio between them will maintain the same for different signal power.

\section{Conclusions}

By analyzing the zoomed-in spectral coherence function of short time signal, distinctive detailed features are observed for different modulations in underwater acoustic communication signals. Taking advantage of such features in short time SOF, we proposed a blind modulation detection algorithm coupled with previously developed blind parameter estimation algorithm for underwater acoustic communication signals. Specifically, the detailed zoomed-in features in SOF is exploited and the ratio between the energy in two different regions in detailed SOF is applied as the metric to distinguish different modulations. Real experimental data collected at sea are used to validate the effectiveness of the proposed algorithm. It is also important to note that the proposed method does not assume any a priori knowledge of the target signal, and the modulation detector does not have power uncertainty problem.

\section{ACKNOWLEDGMENT}

This work is supported by the Office of Naval Research.

\section{REFERENCES}

[1] W. A. Gardner, Cyclostationarity in Communications and Signal Processing. IEEE Press, Piscataway, NJ, USA, 1993.

[2] W. A. Gardner, W. A. Brown, and C.-K. Chen, "Spectral correlation of modulated signals, part II: digital modulation," IEEE Transactions on Communications, vol. 35, no. 6, pp. 595-601, 1987.

[3] W. A. Gardner, "Signal interception: A unifying theoretical framework for feature detection," IEEE Transactions on Communications, vol. 36, no. 8, pp. 897-906, 1988.

[4] A. Fehske, J. Gaeddert, and J. Reed, "A new approach to signal classification using spectral correlation and neural networks," in New Frontiers in Dynamic Spectrum Access Networks, 2005 (DySPAN 2005), pp. $144-150$, nov. 2005.

[5] M. Tsatsanis and G. Giannakis, "Blind estimation of direct sequence spread spectrum signals in multipath," IEEE Transactions on Signal Processing, vol. 45, pp. 1241-1252, May 1997.

[6] S. Buzzi and V. Massaro, "Parameter estimation and multiuser detection for band-limited long-code CDMA systems," IEEE Transactions on Wireless Communications, vol. 7, pp. 2307-2317, June 2008.

[7] W. Gardner, "Exploitation of spectral redundancy in cyclostationary signals," IEEE Signal Processing Magazine, vol. 8, pp. 14-36, Apr. 1991.

[8] E. Like, V. D. Chakravarthy, P. Ratazzi, and Z. Wu, "Signal classification in fading channels using cyclic spectral analysis," EURASIP Journal on Wireless Communications and Networking, no. 1, pp. 1-14, 2009.

[9] T. C. Yang, "Correlation-based decision feedback equalizer for underwater acoustic communications," in IEEE Journal of Oceanic Engineering, vol. 30, pp. 865-880, Oct. 2005.

[10] T. C. Yang and W.-B. Yang, "Performance analysis of direct-sequence spread spectrum underwater acoustic communications with low signalto-noise-ratio input signals," in J. Acoust. Soc Am., vol. 123(2), pp. 842855, Feb. 2008.

[11] G. Eynard and C. Laot, "Performance analysis of direct-sequence spread spectrum underwater acoustic communications with low signal-to-noiseratio input signals," in proc. of the IEEE OCEAN 2008, pp. 1-5, 2008.

[12] T. C. Yang, "Performance analysis of direct-sequence spread spectrum underwater acoustic communications with low signal-to-noise-ratio input signals," in J. Acoust. Soc Am., vol. 124(6), Dec. 2008.

[13] Z. Wu, T. yang, and Z. Liu, "Blind cyclostationary carrier frequency and symbol rate estimation for underwater acoustic communication," IEEE ICC 2012, pp. 3482-3486, 2012.

[14] Z. Wu, T. yang, and Z. Liu, "Modulation detection of underwater acoustic communication signals through cyclostationary analysis," IEEE Milcom 2012, pp. 1-6, 2012. 